\title{
Diagnostic et traitement du déficit en androgènes des patients infectés par le virus de l'immunodéficience humaine
}

\author{
François PRALONG
}

Division d'Endocrinologie, Département de médecine interne, Lausanne, Suisse

\section{RESUME}

La prévalence du déficit en androgènes parmi les hommes atteints par le virus de l'immunodéficience humaine (VIH) varie entre $35 \%$ et plus de $50 \%$. L'hypogonadisme est le plus souvent d'origine centrale, secondaire à la maladie systémique, aux infections opportunistes, à la malnutrition ou parfois également aux traitements administrés. Depuis l'avènement de thérapies anti-rétrovirales efficaces, cette prévalence a diminué mais demeure significative, notamment parmi les patients souffrant d'un syndrome de perte de poids lié au VIH. Lors d'hypogonadisme associé à une perte de poids, il est en outre possible que le déficit en androgènes aggrave la perte de masse maigre. C'est l'une des raisons pour lesquelles il est indiqué de dépister l'hypogonadisme chez tout patient infecté par le VIH et souffrant d'une perte de poids importante.

La substitution hormonale des patients hypogonadiques infectés par le VIH est indiquée, qu'ils soient symptomatiques (diminution de la libido, impuissance) ou pas. En effet, le traitement hormonal améliore la composition corporelle, l'ostéoporose, la qualité de vie et l'humeur, et il induit également une augmentation de la masse corporelle maigre et de la résistance à l'effort de ces patients. Les alternatives de traitement que nous proposons peuvent alors être un schéma d'injections intramusculaires d'esters de testostérone ou l'application quotidienne de préparations transdermiques. Par contre, il n'a pas été démontré que le traitement par des analogues synthétiques de la testostérone du type oxandrolone ou nandrolone induise un effet bénéfique supplémentaire par rapport à la testostérone. Finalement, aucune étude n'a pu met- tre en évidence un quelconque effet bénéfique de l'administration de testostérone à des patients eugonadiques infectés par le VIH et ce même en présence d'un syndrome de perte de poids lié au virus. La substitution hormonale n'est donc pas indiqué en dehors d'un contexte d'hypogonadisme avéré.

Mots-clés : VIH, testostérone, hypogonadisme, perte de poids

\section{INTRODUCTION}

Différents désordres endocriniens sont fréquents chez les personnes infectées par le virus de l'immunodéficience humaine (VIH). Ceux-ci comprennent des altérations des fonctions surrénalienne, thyroïdienne et gonadique qui peuvent être d'origine primaire ou secondaire $[9,12,18]$. Ces différentes altérations peuvent être causées par une variété de facteurs étiologique : à côté des atteintes dues au VIH lui-même, elles peuvent également découler des infections opportunistes secondaires au déficit immunitaire, ainsi que d'éventuels effets secondaires des médicaments dirigés contre le virus ou ces mêmes infections opportunistes.

Il existe notamment une association relativement fréquente entre infection par le VIH et hypogonadisme. La prévalence du déficit en androgène chez les patients infectés

\section{Correspondance :}

Dr François Pralong - Médecin-adjoint, Division d'Endocrinologie, BH 19-709, CHU Vaudois, 1011 Lausanne. Suisse $T e l+41213140619$ - Fax +41213140630 -

Email Francois.Pralong@chuv.hospvd.ch 
par ce virus a été estimée initialement entre $30 \%$ et plus de $50 \%$ selon les études $[9,23]$. L'infection par le VIH peut également se compliquer de changements caractéristiques de la composition corporelle et de l'état nutritionnel des individus affectés, connu sous le nom de syndrome de perte de poids associé au VIH [19]. Le syndrome de perte de poids lié à l'infection VIH semble contribuer de manière importante à l'hypogonadisme associé [7]. De plus, il a été proposé que le déficit en androgènes participe également à l'étiopathogénie de cette perte de poids. Les mécanismes impliqués dans le déficit en androgènes lié au syndrome de perte de poids de l'infection VIH de même que les implications thérapeutiques sont discutés.

\section{HYPOGONADISME ET INFECTION PAR}

\section{LE VIH}

L'épidémie du VIH est apparue de manière rapidement progressive dans les années 1980 . A cette époque, la brutalité de la maladie, son évolution quasi inexorable vers une issue plus ou moins rapidement fatale et l'ignorance dans laquelle nous nous trouvions face à son étiologie rendaient futile l'étude des troubles endocriniens qui lui étaient associés. C'est probablement la raison pour laquelle les premiers rapports sur l'association entre hypogonadisme et infection par le VIH n'apparaissent dans la littérature qu'à la fin des années $1980[4,9]$.

C'est une étude publiée en 1992 qui démontre pour la première fois une évolution quelque peu surprenante des taux circulants de testostérone en fonction de la gravité de l'atteinte due au VIH [4]. En effet, ces résultats montraient que la testostérone plasmatique totale augmente significativement par rapport aux contrôles durant les stades précoces de la maladie (SIDA stades II et III). Dans les stades de SIDA plus tardifs (stades IV-C et IV-D), ces taux diminuaient ensuite pour atteindre des niveaux égaux ou légèrement inférieurs à ceux mesurés chez les contrôles sains [4]. Ces résultats ont été confirmés et expliqués par une autre étude, pour laquelle les patients avaient été stratifiés selon leur taux circulant de lymphocytes CD4 [17]. Dans cette étude également, la testostérone totale était égale ou supérieure à celle des contrôles dans tous les groupes de patients [17]. Par contre, la fraction de testostérone libre évaluée par la mesure de la testostérone non-liée à la SHBG (Sex Hormone Binding Globulin, la protéine transporteuse de testostérone) était significativement diminuée chez tous les patients. Cette diminution corrélait parfaitement avec l'augmentation, dont la cause est demeurée inexpliquée, des concentrations circulantes de SHBG également observée chez les patients infectés par le VIH [17]. De manière remarquable, la diminution des taux de testostérone libre était linéaire et directement corrélée aux taux circulants de lymphocytes CD4, une observation valable également pour la DHEA et l'androstènedione [17]. L'as- sociation existant entre infection par le VIH et hypogonadisme est maintenant une entité clinique bien reconnue, qui mérite d'être dépistée dans certaines situations cliniques comme nous le verrons plus bas, et traitée adéquatement [20].

\section{Mécanismes physiopathologiques}

Le déficit en androgènes associé à l'infection par le VIH est plus fréquemment d'origine secondaire, hypophysaire ou hypothalamique, que primitivement testiculaire [12]. Chez l'homme, le composé androgénique principal est la testostérone, sécrétée par les cellules testiculaires de Leydig. Des atteintes testiculaires d'origine infectieuse, rencontrées lors de formes disséminées d'infections dues à des germes opportunistes tels que Mycobacterium avium intracellulare, Toxoplasma ou CMV, peuvent expliquer une atteinte primitive des cellules de Leydig [2, 8, 21, 26]. De même, l'envahissement testiculaire par des lymphomes ou des syndromes de Kaposi a été décrit $[9,26]$. Finalement, l'infection du testicule par le VIH lui-même pourrait être relativement fréquente [25], ce qui revêt également une importance pour la transmission de la maladie. Ces différentes atteintes testiculaires sont responsables des formes primaires de déficit en androgènes chez les patients infectés par le VIH.

Un certain nombre de médicaments utilisés dans le traitement du SIDA ou de ses complications a également été associé avec un hypogonadisme. Le kétoconazole peut interférer directement avec la stéroïdogenèse et diminuer la synthèse de testostérone [27], induisant par là un hypogonadisme primaire. Par contre, l'acétate de médroxyprogestérone, les glucocorticoïdes et tous les stéroïdes anabolisants réduisent la sécrétion de gonadotrophines hypophysaires, aboutissant ainsi à un hypogonadisme secondaire [20].

Toutes causes étant confondues, le déficit en androgènes des patients infectés par le VIH correspond à un hypogonadisme hypogonadotrope dans plus de $75 \%$ des cas. Bien que des infections opportunistes du système nerveux central ne soient pas rares chez ces patients, cet hypogonadisme survient généralement en l'absence de lésion de la région hypothalamo-hypophysaire. Il s'explique par les répercussions hypothalamiques de la maladie systémique, souvent associée à une malnutrition et/ou une perte de poids. L'association entre le syndrome de perte de poids liée à l'infection par le VIH et un hypogonadisme hypogonadotrope représente d'ailleurs une sorte de cercle vicieux. En effet, il est bien établi qu'une perte de poids, lorsque elle est significative, peut inhiber l'axe gonadotrope neuroendocrinien [22]. D'autre part, les androgènes sont des hormones à activité anabolisante (et probablement également stimulante de la prise alimentaire). Un déficit en androgènes peut donc potentiellement aggraver une perte 
de poids, qui va elle-même contribuer à aggraver l'hypogonadisme. Dans ce contexte physiopathologique, il peut devenir très difficile de distinguer la cause de l'effet. Cette relation bi-directionnelle entre hypogonadisme ef balance énergétique négative peut en tous les cas partiellement expliquer l'association existant entre hypogonadisme et perte de poids liée au VIH.

\section{Le syndrome de perte de poids liée au VIH}

La perte de poids lors de l'infection par le VIH peut devenir chez un certain nombre de patients un problème clinique important, contribuant de manière significative à la morbidité liée à cette infection. Le syndrome est défini par le CDC américain (Centers for Disease Control) comme une perte de plus de $10 \%$ du poids corporel initial, en l'absence d'une autre étiologie évidente que l'infection par le VIH (tels que par exemple une infection opportuniste ou un lymphome) [6]. Avant l'ère des thérapies anti-rétrovirales hautement efficaces, sa prévalence était de l'ordre de $70 \%$ [5]. Ce problème demeure cliniquement significatif malgré l'introduction de ces nouvelles thérapies, mais sa prévalence a nettement diminué.

Lorsque la perte de poids est aiguë, elle est fréquemment liée à des infections opportunistes, qui doivent donc être suspectées et recherchées. En revanche, lorsque cette perte de poids revêt un caractère plus chronique, lentement évolutif, elle est le plus souvent liée à l'infection par le VIH lui-même. Elle peut alors constituer une condition dévastatrice, grevée d'une mortalité importante et par là-même revêtir une importance pronostique pour ces patients [3, 16]. En effet, la malnutrition à laquelle aboutit la perte de poids peut interférer de différentes manières avec la lutte de l'organisme contre l'infection par le VIH. Dans un premier temps, il a été suggéré que des déficits de certains micronutriments particuliers pourraient altérer spécifiquement certains aspects spécifiques de la réponse immunitaire. En outre, lorsque la malnutrition devient sévère, on assiste à une baisse plus généralisée de la réponse immunitaire, ce qui revêt évidemment un aspect extrêmement délétère dans le contexte clinique de ces patients.

Comme indiqué plus haut, il a été suggéré que le déficit en androgènes pourrait participer à l'étiopathogénie de la perte de poids liée à l'infection par le VIH. Cette hypothèse a été évaluée par une étude cas-témoin pratiquée sur 26 hommes infectés par le VIH [10] et faisant partie de la "Multicenter AIDS Cohort Study" américaine. Treize sujets présentant une perte de poids liée au VIH selon les critères du CDC furent identifiés, ainsi que 13 contrôles appariés pour l'âge et la durée de suivi au sein de la cohorte mais ne présentant pas de perte de poids significative. Pour les sujets avec perte de poids, un bilan hormonal fut pratiqué sur les échantillons sanguins prélevés lors de l'inclusion, lors du contrôle précédant immédiatement l'identi- fication du syndrome de perte de poids corporel et lors du contrôle au cours duquel le syndrome de perte de poids avait été diagnostiqué. Pour les contrôles, le bilan hormonal fut pratiqué sur l'échantillon prélevé lors de l'inclusion, et sur deux échantillons appariés pour les intervalles de suivi par rapport aux cas précédents.

Les auteurs [10] ont rapporté une baisse significative de la testostérone biodisponible lors de la visite précédant l'identification de la perte de poids chez ces patients, diminution confirmée lors de la troisième visite où les valeurs de testostérone totale et libre étaient également diminuées. Chez les contrôles, les valeurs de testostérone totale, libre et biodisponible n'ont pas varié significativement entre ces trois visites. La diminution de la testostéronémie chez les patients perdant du poids ne s'accompagnait pas d'une augmentation des taux circulants de LH, indiquant une participation centrale au déficit. Une discrète augmentation parallèle des valeurs de FSH indiquait toutefois également une possible atteinte testiculaire primaire au niveau des tubes séminifères chez ces même patients [10].

L'importance clinique de cette observation est démontrée par une autre étude publiée la même année [13]. Cette étude a évalué la résistance physique, en fonction du nombre maximum de passages de la station assise à la station debout durant dix secondes, chez 15 patients infectés par le VIH et présentant un hypogonadisme associé à un syndrome de perte de poids. Les résultats ont montré que la résistance physique était directement corrélée à la testostéronémie [13]. Dans la même étude, les auteurs ont d'autre part pu montrer sur une quarantaine de patients que le déficit en androgènes était également associé à une perte excessive de masse maigre par rapport à la perte de poids totale [13]. Ensemble, ces deux études $[10,13]$ indiquent d'une part que la diminution des taux circulants d'androgènes peut précéder le syndrome de perte de poids, et d'autre part que ce déficit pourrait aggraver la perte relative de masse maigre. Finalement, la seconde étude indique également que le déficit en androgène peut avoir des répercussions fonctionnelles cliniquement mesurables et potentiellement utiles.

\section{Hypogonadisme et VIH après l'avènement des thé- rapies antirétrovirales hautement efficaces}

En raison de l'incidence élevée d'hypogonadisme associé à l'infection par le VIH $[9,12]$, son dépistage est entré dans la pratique quotidienne de la plupart des centres suivant ces patients au long cours. Cette incidence a toutefois diminué depuis l'introduction de thérapies anti-rétrovirales efficaces. Dans une étude récente conduite sur une centaine de patients recevant un traitement anti-rétroviral, Rietschel et al. ont rapporté que les taux moyens de testostérone n'étaient pas différents entre le groupe de patients présentant une perte de poids et ceux n'ayant pas de perte de poids significative [24]. Une analyse plus fine a toutefois 
montré que $5 \%$ des patients avec syndrome de perte de poids avaient des taux circulants de testostérone totale diminués, ce pourcentage augmentant à $19 \%$ lorsqu'on mesurait la testostéronémie libre. Cette observation est probablement utile cliniquement, puisque plus de $80 \%$ des patients hypogonadiques de cette étude se plaignaient de baisse de la libido, alors que ce symptôme n'était présent que chez $47 \%$ des patients eugonadiques [24]. Il faut également souligner qu'il n'y avait aucune corrélation dans cette étude entre le type de traitement reçu par les patients et les taux de testostérone totale ou libre mesurés, démontrant l'absence d'effet de classe des différentes thérapies anti-rétrovirales sur l'hypogonadisme.

Cette observation indique que l'hypogonadisme doit être dépisté chez les patients présentant un syndrome de perte de poids lié au VIH, et ce même en présence d'un traitement anti-rétroviral et d'une immunité relativement préservée. Etant donné le retentissement fonctionnel du déficit en androgènes des patients infectés par le VIH, il est maintenant acquis que ces patients doivent être substitués correctement.

\section{Considérations thérapeutiques}

Les buts visés par la substitution hormonale en androgènes de patients infectés par le VIH peuvent être schématisés ainsi : amélioration de symptômes spécifiques tels que diminution de la libido ou impuissance ; amélioration de symptômes non spécifiques, tels que fatigue et baisse de la force et de l'énergie ; correction de la perte de poids et de l'état catabolique liés à l'infection par le VIH. En fonction de ces buts, il est donc recommandé de traiter les patients hypogonadiques, qu'ils soient symptomatiques ou non [20]. En effet, la substitution hormonale a le potentiel d'améliorer ces sujets d'un point de vue métabolique, comme l'a démontré une étude prospective randomisée et contrôlée par placebo de la substitution en testostérone de patients hypogonadiques infectés par le VIH [14]. Dans cette étude, la substitution en testostérone a permis d'augmenter la masse maigre de ces patients de 2 kilogrammes en moyenne sur six mois, alors que les patients du groupe traité par placebo perdaient 0,6 kilogramme de masse maigre dans le même temps. D'autre part, la substitution a permis d'améliorer la qualité de la vie de ces patients, sans être grevée d'effets secondaires indésirables à la dose de $300 \mathrm{mg}$ de testostérone administrée intra-musculairement toutes les trois semaines [14]. Une autre étude récente du même groupe menée sur un petit collectif de patients a montré que ces effets anabolisants de la testostérone sont maintenus sur le long terme (un an de suivi) [15]. Il existe finalement quelques données suggérant que la substitution en testostérone permet d'améliorer la fonction musculaire de patients hypogonadiques infectés par le VIH [1].
Il n'existe par contre aucune indication à traiter les patients eugonadiques, même lorsqu'ils présentent une perte de poids liée au VIH, par de la testostérone. Les évidences de la littérature suggérant que l'adjonction de testostérone ou d'anabolisants androgéniques à doses pharmacologiques peut améliorer l'état catabolique de ces patients sont encore éparses [11], et l'on s'exposerait alors à des effets secondaires indésirables du traitement sans bénéfice prouvé.

Le composé le plus utilisé pour la substitution des patients hypogonadiques infectés par le VIH reste la testostérone, qui peut être administrée sous la forme d'injections intramusculaires ou par voie trans-cutanée. Par contre, bien que les analogues synthétiques tels que la nandrolone ou l'oxandrolone aient été étudiés chez ces patients, ces composés ont le potentiel d'effets secondaires importants, notamment au niveau hépatique. Comme ils ne sont pas plus efficaces que la testostérone elle-même, leur usage ne peut pas être recommandé. D'autre part, il conviendra de se souvenir dans ce contexte clinique également des contre-indications habituelles à la substitution par la testostérone (cancer de la prostate, hypertrophie bénigne de la prostate, polycythemia vera).

\section{CONCLUSION}

Le déficit en androgènes demeure une affection fréquente chez les patients infectés par le VIH, même depuis l'introduction des thérapies anti-rétrovirales hautement efficaces. Ce déficit peut avoir des conséquences cliniques importantes, et notamment semble participer au catabolisme observé dans le syndrome de perte de poids liée à l'infection par le VIH. En conséquence, son dépistage ainsi que son traitement sont formellement indiqués chez ces patients. Le remplacement hormonal par des formes injectables de testostérone demeure la clé de voûte du traitement de substitution, en raison de son innocuité et de son efficacité bien établies à doses substitutives.

\section{REFERENCES}

1. BHASIN S., STORER T.W., JAVANBAKHT M. et al. : Testosterone replacement and resistance exercise in $\mathrm{HIV}$-infected men with weight loss and low testosterone levels. J. Am. Med. Ass., $2000,283: 763-770$.

2. CHABON A.B., STENGER R.J., GRABSTALD H. : Histopathology of testis in acquired immune deficiency syndrome. Urology, 1987, $29: 658-663$.

3. CHLEBOWSKI R.T., GROSVENOR M.B., BERNHARD N.H., MORALES L.S., BULCAVAGE L.M. : Nutritional status, gastrointestinal dysfunction, and survival in patients with AIDS. Am. J. Gastroenterol., 1989, 84 : 1288-1293.

4. CHRISTEFF N., GHARAKHANIAN S., THOBIE N., ROZENBAUM W., NUNEZ E.A. : Evidence for changes in adrenal and 
testicular steroids during HIV infection. J. Acquir. Immune Defic. Syndr., 1992, 5 : 841-846.

5. CORCORAN C., GRINSPOON S. : Treatments for wasting in patients with the acquired immunodeficiency syndrome. N. Engl. J. Med., 1999, $340: 1740-1750$.

6. Council of State and Territorial Epidemiologists, AIDS Program, Center for Infectious Diseases, Centers for Disease Control. Revision of the CDC surveillance case definition for acquired immunodeficiency syndrome. MMWR, 1987, 36 (suppl 1) : 3S$15 S$.

7. DANOFF A. : Endocrinologic complications of HIV infection. Med. Clin. North Am., 1996, 80 : 1453-1469.

8. DE PAEPE M.E., WAXMAN M. : Testicular atrophy in AIDS: a study of 57 autopsy cases. Hum. Pathol., 1989, 20 : 210-214.

9. DOBS A.S., DEMPSEY M.A., LADENSON P.W., POLK B.F. : Endocrine disorders in men infected with human immunodeficiency virus. Am. J. Med., 1988, 84 : 611-616.

10. DOBS A.S., FEW W.L., 3RD, BLACKMAN M.R., HARMAN S.M., HOOVER D.R., GRAHAM N.M. : Serum hormones in men with human immunodeficiency virus-associated wasting. J. Clin. Endocrinol. Metab., 1996, 81 : 4108-4112.

11. DOBS A.S. : Is there a role for androgenic anabolic steroids in medical practice ? J. Am. Med. Ass., 1999, 281 : 1326-1327.

12. GRINSPOON S.K., BILEZIKIAN J.P. : HIV disease and the endocrine system. N. Engl. J. Med., 1992, 327 : 1360-1365.

13. GRINSPOON S., CORCORAN C., LEE K. et al. : Loss of lean body and muscle mass correlates with androgen levels in hypogonadal men with acquired immunodeficiency syndrome and wasting. J. Clin. Endocrinol. Metab., 1996, 81 : 4051-4058.

14. GRINSPOON S., CORCORAN C., ASKARI H. et al. : Effects of androgen administration in men with the AIDS wasting syndrome. A randomized, double-blind, placebo-controlled trial. Ann. Intern. Med., 1998, 129 : 18-26.

15. GRINSPOON S., CORCORAN C., ANDERSON E. et al. : Sustained anabolic effects of long-term androgen administration in men with AIDS wasting. Clin. Infect. Dis., 1999, 28 : 634-636.

16. KOTLER D.P., TIERNEY A.R., WANG J., PIERSON R.N. JR. : Magnitude of body-cell-mass depletion and the timing of death from wasting in AIDS. Am. J. Clin. Nutr., 1989, 50 : 444-447.

17. LAUDAT A., BLUM L., GUECHOT J. et al. : Changes in systemic gonadal and adrenal steroids in asymptomatic human immunodeficiency virus-infected men : relationship with the CD4 cell counts. Eur. J. Endocrinol., 1995, 133 : 418-424.

18. MERENICH J.A., MCDERMOTT M.T., ASP A.A., HARRISON S.M., KIDD G.S. : Evidence of endocrine involvement early in the course of human immunodeficiency virus infection. J. Clin. Endocrinol. Metab., 1990, 70 : 566-571.

19. MHIRI C., BELEC L., DI COSTANZO B., GEORGES A., GHERARDI R. : The slim disease in African patients with AIDS. Trans. R. Soc. Trop. Med. Hyg., 1992, 86 : 303-306.

20. MYLONAKIS E., KOUTKIA P., GRINSPOON S. : Diagnosis and treatment of androgen deficiency in human immunodeficiency virus-infected men and women. Clin. Infect. Dis., 2001, 33 : 857-864.
21. PORETSKY L., CAN S., ZUMOFF B. : Testicular dysfunction in human immunodeficiency virus-infected men. Metabolism, 1995, 44 : 946-953.

22. PRALONG F.P., CASTILLO E., RAPOSINHO P.D., AUBERT M.L., GAILLARD R.C. : Obesity and the reproductive axis. Ann. Endocrinol. (Paris), 2002, 63 : 129-134.

23. RAFFI F., BRISSEAU J.M., PLANCHON B., REMI J.P., BARRIER J.H., GROLLEAU J.Y. : Endocrine function in $98 \mathrm{HIV}-$ infected patients : a prospective study. Aids, 1991, $5: 729-733$.

24. RIETSCHEL P., CORCORAN C., STANLEY T., BASGOZ N., KLIBANSKI A., GRINSPOON S. : Prevalence of hypogonadism among men with weight loss related to human immunodeficiency virus infection who were receiving highly active antiretroviral therapy. Clin. Infect. Dis., 2000, 31 : 1240-1244.

25. SHEVCHUK M.M., NUOVO G.J., KHALIFE G. : HIV in testis: quantitative histology and HIV localization in germ cells. J. Reprod. Immunol., 1998, 41 : 69-79.

26. SHEVCHUK M.M., PIGATO J.B., KHALIFE G., ARMENAKAS N.A., FRACCHIA J.A. : Changing testicular histology in AIDS : its implication for sexual transmission of HIV. Urology, 1999, 53 : 203-208.

27. VAN TYLE J.H. : Ketoconazole. Mechanism of action, spectrum of activity, pharmacokinetics, drug interactions, adverse reactions and therapeutic use. Pharmacotherapy, 1984, $4: 343-373$.

\section{ABSTRACT}
Diagnosis and treatment of androgen deficiency in patients with human immune deficiency virus infection

\section{François PRALONG}

Androgen deficiency is frequent among men infected by the human immune deficiency virus (HIV), with an estimated prevalence of between $35 \%$ and $50 \%$. Primary testicular damage has been described, either due to the virus itself, opportunistic agents such as CMV, Toxoplasma gondii or Mycobacterium avium intracellulare, or less frequently neoplastic invasion by lymphoma or in a context of Kaposi's sarcoma. However, secondary hypogonadism remains a more frequent cause. Hypogonadotropic hypogonadism can be secondary to opportunistic infections, malnutrition, and sometimes even certain therapeutic agents. Since the introduction of highly active antiretroviral therapies, the prevalence of hypogonadism has substantially decreased. However, it remains a significant clinical problem, particularly among patients suffering from wasting, as androgen deficiency may aggravate the loss of lean body mass observed in the wasting syndrome 
of HIV patients. Screening for androgen deficiency is therefore indicated in HIV patients suffering from wasting, even in the absence of specific symptoms.

Androgen replacement therapy is justified in symptomatic (loss of libido, impotence) and asymptomatic patients with documented hypogonadism. We recommend replacement therapy with testosterone by subcutaneous or intramuscular injection. In the absence of specific symptoms, it should be remembered that testosterone replacement therapy of HIVinfected hypogonadic patients is associated with improvements in body composition and muscle strength, bone densitometry, quality of life and mood. Similar improvements have also been demonstrated in hypogonadic patients with wasting syndrome. Synthetic testosterone analogues such as oxandrolone or nandrolone do not seem to be more powerful than testosterone at replacement doses, and may be associated with more side effects, particularly severe hepatic dysfunction. In contrast, there is no proven benefit of androgen treatment of eugonadic HIV-infected patients, and the treatment of such patients with androgens, even in the presence of wasting, cannot be recommended.

Key words: HIV, testosterone, hypogonadism, weight loss

Communication au $\mathrm{XIX} \mathrm{X}^{\circ}$ Congrès de la Société d'Andrologie de Langue Française, Genève, 12-14 décembre 2002.

Manuscrit reçu : avril 2003 ; accepté juin 2003. 